\title{
Erratum
}

European J. Appl. Microbiol. Biotechnol. 5, 13-16 (1978):

\section{Immobilization of Glycolysis System of Yeasts and Production of Cytidine Diphosphate Choline}

\author{
Akira Kimura ${ }^{1}$, Yoshinori Tatsutomi ${ }^{2}$, Naoki Mizushima ${ }^{2}$, Atsuo Tanaka ${ }^{3}$, \\ Ryu'ichi Matsuno ${ }^{2}$, and Hirosuke Fukuda ${ }^{1}$ \\ ${ }^{1}$ The Research Institute for Food Science, Kyoto University, Uji, Kyoto 611, Japan \\ 2 Department of Food Science and Technology, Faculty of Agriculture, Kyoto University, \\ Kyoto 606, Japan \\ 3 Department of Industrial Chemistry, Faculty of Engineering, Kyoto University, Kyoto 606 , \\ Japan
}

On page 13 the structural formula should have been labeled Figure 1. The legend should have read: According to the number of $n$, various polymerized compounds with different average molecular weight (M.W.) were prepared, for example, average M. W. $1000,2000,4000$, and 6000 , but only M. W. 1000 was good for entrapping the whole enzyme system

Figure 1 on page 14 should have been Figure 2.

The second sentence in the last paragraph on page 14 should have read: Only the smallest one (average $M . W .=1000$ ) effectively entrapped whole enzymes as shown in Figure 3.

The figure at the top of page 15 should have been Figure 3 . The legend should have read: The reaction was carried out by the cells immobilized in ENT of M. W. 1000. After 3, 6, and $9 \mathrm{~h}$ in the first reaction, the immobilized cells were separated by centrifugation, washed 3 times with deionized water and reused in the fresh reaction mixture containing $2 \mathrm{mM}$ ATP, and $2 \mathrm{mM}$ NAD in addition to the usual components (although ATP was not always necessary).

The figure at the bottom of page 15 should have been Figure 4 .

The legend should have read: The immobilized cells were kept at $4^{\circ} \mathrm{C}$ for the days indicated $(2,7$, and 14 days), then the cells were used as described in the legend for Figure 3 . The cells were reused after $3 \mathrm{~h}$ reaction 